\title{
Correction: The Characteristics and Risk Factors of Web-Based Sexual Behaviors Among Men Who Have Sex With Men in Eastern China: Cross-sectional Study
}

Lin Chen, MD; Wanjun Chen, MD; Tingting Jiang, MD; Zhikan Ni, MD; Qiaoqin Ma, College Degree of Medicine; Xiaohong Pan, MD

Zhejiang Provincial Center for Disease Control and Prevention, Hangzhou, China

\section{Corresponding Author:}

Xiaohong Pan, MD

Zhejiang Provincial Center for Disease Control and Prevention

3399 Binsheng Road, Binjiang District

Hangzhou, 310051

China

Phone: 86057187115191

Email: xhpan@cdc.zj.cn

\section{Related Article:}

Correction of: https://publichealth.jmir.org/2021/9/e25360

(JMIR Public Health Surveill 2021;7(9):e33430) doi: 10.2196/33430

In "The Characteristics and Risk Factors of Web-Based Sexual Behaviors Among Men Who Have Sex With Men in Eastern China: Cross-sectional Study" (JMIR Public Health Surveill 2021;7(9):e25360), one error was noted.

Due to a system error, the name of one author, Lin Chen, was replaced with the name of another author on the paper, Xiaohong Pan. In the originally published paper, the order of authors was listed as follows:

Xiaohong Pan, Wanjun Chen, Tingting Jiang, Zhikan Ni, Qiaoqin Ma, Xiaohong Pan

This has been corrected to:

Lin Chen, Wanjun Chen, Tingting Jiang, Zhikan Ni, Qiaoqin Ma, Xiaohong Pan
In the originally published paper, the ORCID of author Lin Chen was incorrectly published as follows:

$$
\text { 0000-0003-3373-3393 }
$$

This has been corrected to:

$$
\text { 0000-0003-2197-2733 }
$$

The correction will appear in the online version of the paper on the JMIR Publications website on September 8, 2021, together with the publication of this correction notice. Because this was made after submission to PubMed, PubMed Central, and other full-text repositories, the corrected article has also been resubmitted to those repositories.

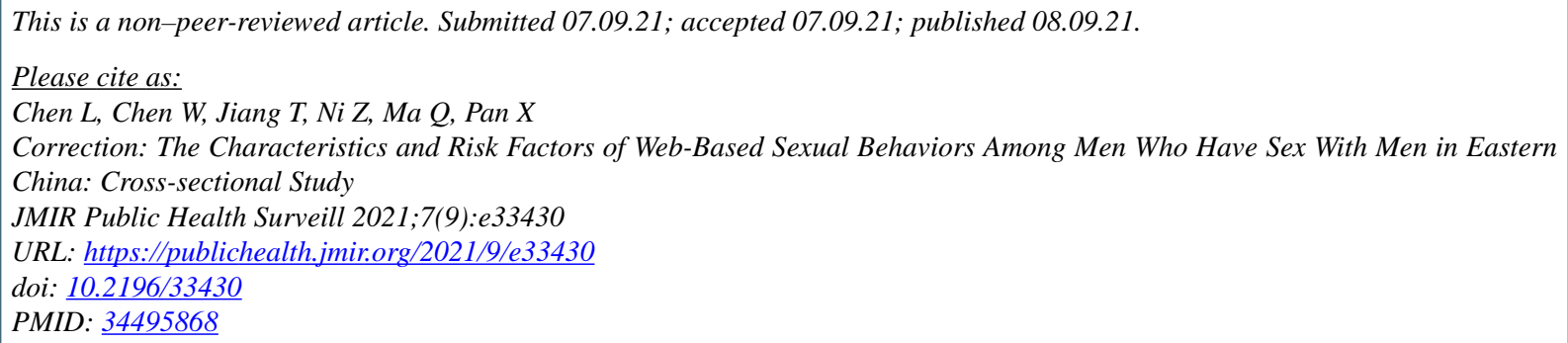

CLin Chen, Wanjun Chen, Tingting Jiang, Zhikan Ni, Qiaoqin Ma, Xiaohong Pan. Originally published in JMIR Public Health and Surveillance (https://publichealth.jmir.org), 08.09.2021. This is an open-access article distributed under the terms of the Creative Commons Attribution License (https://creativecommons.org/licenses/by/4.0/), which permits unrestricted use, distribution, 
and reproduction in any medium, provided the original work, first published in JMIR Public Health and Surveillance, is properly cited. The complete bibliographic information, a link to the original publication on https://publichealth.jmir.org, as well as this copyright and license information must be included. 